CASE REPORTS

\title{
PENETRATING INJURY EXTENDING FROM ORAL CAVITY TO THE NECK - WITH AN INDIGENOUS WEAPON
}

\author{
TALUKDER DC ${ }^{1}$, ISLAM MZ ${ }^{2}$, KHAN MAS $^{3}$, RAHMAN A ${ }^{4}$
}

\begin{abstract}
:
A penetrating injury that extends from oral cavity deep into the right side of the neck. It is a diagnostic and a therapeutic challenge to the treating surgeon, because a number of important structures are densely packed in a small area, and the injury to any of these structures may immediately cause a life threatening situation. We are describing an interesting case, where a 55- year old man presented with a penetrating neck injury which was caused by an indigenous weapon, which ran from the oral cavity to the right side of the neck, $X$ ray revealed the elongated pointed tip foreign object ran through oral cavity to the level of the body of $7^{\text {th }}$ cervical vertebrae. The patient was found to have stable vitals and no active bleeding from the wounds; exploration of the neck was done under General aneasthesia with endo tracheal intubation through tracheostomy opening. The weapon was dislodged from the bamboo made handle, and retrieved under direct vision. Surprisingly, there were no injuries to any vital structures of the neck. The patient made an uneventful recovery.
\end{abstract}

Key words: Penetrating injury; oral cavity ; elongated Foreign body.

J Dhaka Med Coll. 2013; 22(1) : 87-89.

\section{Introduction}

Penetrating neck injuries represent approximately $5 \%-10 \%$ of all the trauma cases ${ }^{1}$. Mostly they are from fire arm or stab injuries. Though the course of the stab wounds may be more limited than that of gunshot wounds, in the neck, number of vital structures are densely packed and so a stab injury can still have a clear potential to cause one or multiple major structural damages. The management should start with the Advanced Trauma Life Support (ATLS) protocol. Necessary investigations along with prompt medical and surgical therapies would minimize the morbidity and the mortality which are associated with such injuries.

\section{Case Report}

A 55-year-old male agricultural worker from Hobiganj presented with a history of physical assault followed by penetrating injury through the floor of the oral cavity to the right side of the neck 6 hours back. This was caused by an indigenous weapon so called Totta while he was lying in his house. He was conscious and oriented; his pulse rate was $96 /$ minute with a $\mathrm{BP}$ of $100 / 65 \mathrm{~mm}$ of $\mathrm{Hg}$. The 1st wound which was in the floor of oral cavity in right side, size is about $1.5 \times 1.5 \mathrm{~cm}$ mostly obscured with its bamboo made handle, and the weapon's tip had penetrated through this wound. 2nd wound was of size $2 \times 2 \mathrm{~cm}$ seen over the right side of the neck, just lateral to the cricoid cartilage. This was a hard swelling in the right, but it did not expand in size or pulsate. He complained of severe pain in the neck during any neck movement and talking or swallowing. The pulsation of the carotid, superficial temporal, brachial and the radial arteries was intact and it was symmetrical bilaterally. On neurological examination no abnormality was seen. The expansion of the chest and the bilateral air entry in the chest were normal. No oronasopharyngeal bleeding, no active bleeding

1. Dr. Debesh Chandra Talukder, Assistant Professor \& RS, Department of ENT , Dhaka Medical College Hospital, Dhaka

2. Dr. MZ Islam, Indoor Medical Officer ENT Oncology, NICRH Mohakhali, Dhaka

3. Dr. MAS Khan, RS, Department of Surgery, Dhaka Medical College Hospital, Dhaka

4. Dr. Atiar Rahman, Assistant Professor, Department of Surgery, DMCH, Dhaka

Correspondence : Dr. Debesh Chandra Talukder, FCPS ENT, Assistant Professor \& RS, Department of ENT, Dhaka Medical College Hospital, Dhaka 


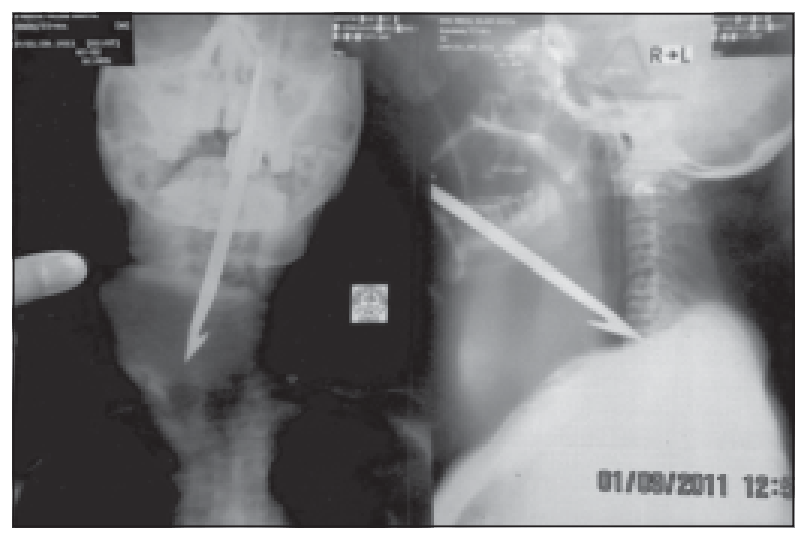

Fig.-1: $X-$ Ray soft tissue neck $A / P$ and lateral view

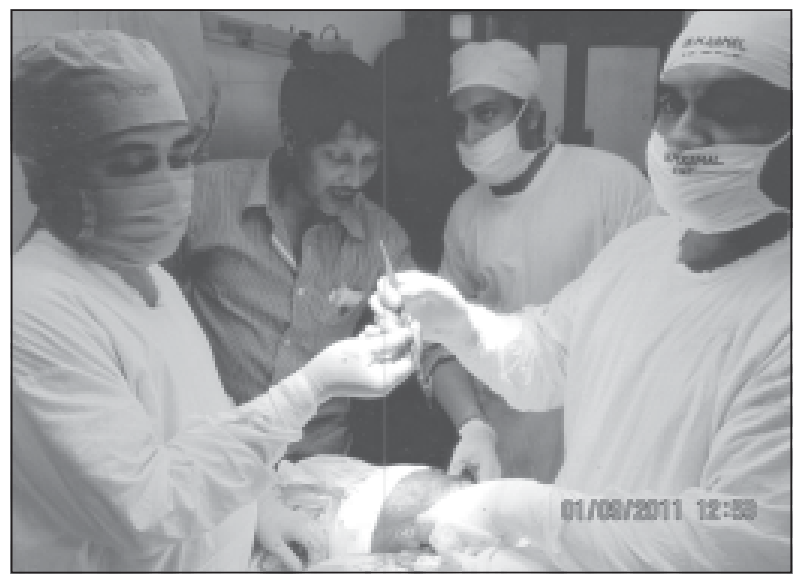

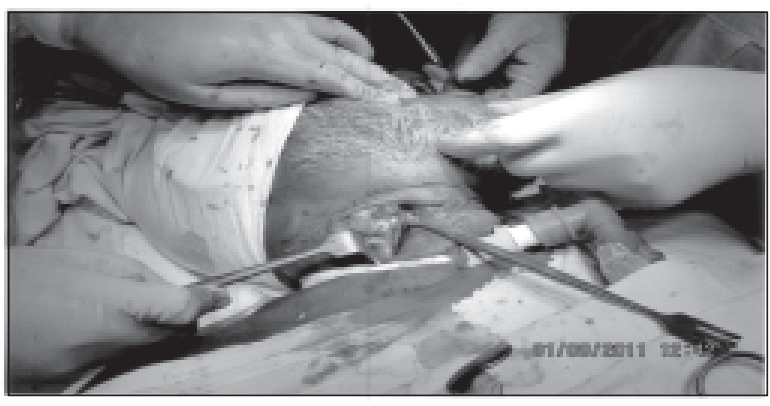

Fig.-2: Horizontal incision was made over the swelling of the Neck tip of the weapon seen

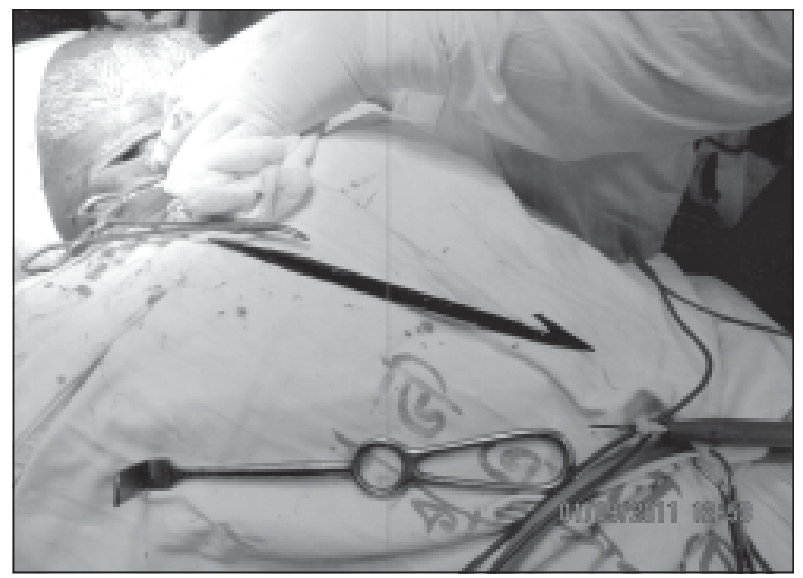

Fig.-3 : Foreign body removed

from the wound, no expanding haematoma and no subcutaneous emphysema were seen. The patient was started on intravenous fluids and was given antibiotics, and tetanus prophylaxis. A blood specimen was collected for haemogram, blood grouping and blood chemistry studies. The cervical antero-posterior and lateral radiography showed a pointed elongated foreign object having an arched in its tip (Figure1) which penetrated from the right side of the oral cavity, which directed laterally and downwards; crossed the midline. Tracheostomy done earlier then exploration of the neck was done under general anaesthesia with endotracheal intubation through tracheostomy opening.

An horizontal incision which was made directly over the swelling (Fig.-2) .On dissection, the weapon was found to pass below the right sterno-clidomastoid and the curved tip was found to point towards the swelling .The weapon was then gently dislodged from the handle by saw and retrieved under direct vision through the incision made over the swelling. (Fig.-3) After the removal of the weapon, their was no active bleeding. And all the major structures on either side of it were found to be uninjured. Both the wound of right side of the neck and the floor of the mouth were thoroughly cleaned and closed in 2 layers keeping a drain in situ. Intra-operative laryngoscopy, and oesophagoscopy were done by using a rigid endoscope. revealed normal findings.Postoperatively, the patient was continued on IV fluids, intravenous antibiotics and analgesics. The drain was removed on the third postoperative day. The patient had an uneventful recovery.

\section{Discussion}

The management of a penetrating neck injury should start with the Advanced Trauma Life Support (ATLS) protocol. After the stabilization 
of the patients' condition, the workup should proceed in a timely manner An immediate exploration is warned against, in the presence of an active bleeding with signs and symptoms of shock, or an expanding haematoma ${ }^{2}$. Probing or local exploration of the neck to remove the foreign body should not be attempted in the emergency department, because the injuries in the major vessels may be tamponated by foreign bodies or a clot. Therefore, the blind removal of the objects may dislodge the clot and may initiate a life threatening haemorrhage ${ }^{3}$. In stable patients, preliminary laboratory tests like evaluation of haemoglobin and the haematocrit level to determine the degree of blood loss, that of the glucose and electrolyte levels and toxicologic screening are also carried out ${ }^{5}$. A chest and neck radiograph is very useful to evaluate the position of the foreign body; any vertebral injury; and free air in the prevertebral or the deep neck spaces, which are suggestive of an aerodigestive tract injury. For analyzing wounds based on their craniocaudal locations, the neck is divided into 3 zones by using anatomic landmarks ${ }^{4}$. Each zone has a group of vital structures that can be injured and this zonal division may determine the kind of trauma management which has to be given. Zone-I is the horizontal area between the clavicle/suprasternal notch and the cricoid cartilage; Zone-II is the area between the cricoid cartilage and the angle of the mandible; Zone-III is the area between the angle of the mandible and the base of the skull In our case, there was no clinical sign of any vascular injury and the X-ray findings were not suggestive of any injury to the aerodigestive tract. So we decided to explore the neck without any further delay. We explored the neck because firstly, on its long path, the weapon had a clear potential to injure many structures; secondly, to confirm the nature of the swelling whether it was a haematoma or the projected tip of the weapon or anything else; and lastly, On dissection, the swelling was found to be the projected tip of the weapon and not a haematoma and the important structures was also found to be uninjured. If an aerodigestive tract injury is suspected clinically before the neck exploration or if it is found during the exploration, intraoperative triple endoscopy (laryngoscopy, bronchoscopy, and oesophagoscopy) should be performed because the early diagnosis and the management of the oesophageal injuries decreases the chances of devastating complications like mediastinitis ${ }^{6}$. So, with a high suspicion of aero-digestive tract injury, we performed an endoscopy and the findings were normal. Postoperatively, the patients were monitored closely in intensive care units with serial examinations to identify any missed injuries. Par-enteral broad-spectrum antibiotics are the standard treatment and if no evidence of leak is present, drains and feeding tubes are discontinued and oral feeding is started

\section{Conclusion :}

To conclude, in a penetrating neck injury, where a large pointed foreign body enters the oral cavity and points on the right side of the neck, exploration of the neck should be done without further delay, because it gives the best opportunity to assess the whole injury tract visually and to manage such injuries properly.

\section{References}

1. Alterman DM, Daley BJ, Cheng EU, Selimanon V. Penetrating neck trauma. Medscape. [online] 2008 Sept. 9. available from: URL:http// emedicine.medscape.com/article/433306.

2. Tuncyurek P, Cabbarpur C, Aksu H. A case report on a foreign body which caused a penetrating neck injury. Ulusal Travma Dergisi. 2001; 7: 204-06.

3. Peloponissios N, Halkic N, Moeschler O, Schnyder $\mathrm{P}$, Vuilleumier H. Penetrating thoracic trauma in arrow injuries. Ann Thorac Surg. 2001; 71 : 1019-21.

4. Saletta JD, Folk FA, Freeark RJ. Trauma to the neck region. Surg Clin North Amer. 1973; 53: 73-86.

5. Demetriades D, Theodorou D, Cornwell E, Berne $\mathrm{TV}$, Asensio J, Belzberg $\mathrm{H}$, et al. Evaluation of penetrating injuries on the neck: a prospective study on 223 patients. World J Surg. 1997; 21: 41-47.

6. Singh RK, Bhandary S, Karki P. Managing a wooden foreign body in the neck. J Emerg Trauma Shock. 2009; 2(3):191-95. 